\title{
Analgesic Efficacy and Safety of Nonsteroidal Anti-Inflammatory Drugs after Transurethral Resection of Prostate
}

\author{
Cengiz Kara, Berkan Resorlu, Izzet Cicekbilek, Ali Unsal
}

Department of Urology, Kecioren Training and Research Hospital, Ankara, Turkey

\begin{abstract}
Objectives: The aim of this study was to assess the analgesic efficacy and safety of nonsteroidal anti-inflammatory drugs (NSAIDs), administered as intramuscular diclofenac in comparison with intravenous paracetamol after transurethral resection of the prostate (TURP).

Materials and Methods: Fifty men, aged 55 to 75 years, undergoing TURP at our hospital were included in this study. Patients were divided randomly and prospectively into two groups (25 patients in each group). Group I (NSAID) received 75 mg of diclofenac i.m. at the end of the operation followed by $75 \mathrm{mg}$ of diclofenac i.m. for 24 hours $(75 \mathrm{mg} \times 2$ once a day $=150$ $\mathrm{mg} / 24 \mathrm{~h}$ ) postoperatively. The other group (Group II) consisted of patients who received $1 \mathrm{~g} / 100 \mathrm{~mL}$ i.v. paracetamol 15 minutes twice daily as postoperative analgesia. Postoperative pain scores were evaluated at 30 minutes, 1, 2, 4 and 6 hours after administration of each analgesic, using a visual analogue scale (VAS). Furthermore, preoperative and postoperative hemoglobin $(\mathrm{Hb})$ levels and hemostatic variables (bleeding time, prothrombine time and the international normalized ratio, i.e. the ratio of a patient's prothrombin time to a normal [control] sample) were recorded in all patients.

Results: The pain score changes during a 4 hour period between the two groups was similar $(p=0.162)$. Thirty minutes after surgery, pain scores were high $(>3 \mathrm{~cm})$ in both groups and without differences between groups $(\mathrm{p}=0.11)$ but 6 hours after surgery, pain scores were significantly higher with paracetamol compared to diclofenac $(\mathrm{p}<0.05)$. No significant difference was observed between the groups regarding the amount of resected tissue, operating time, preoperative-postoperative $\mathrm{Hb}$ levels and hemostatic variables. In the both groups, no patient required blood transfusion postoperatively.

Conclusions: NSAIDs are not a contraindication to TURP and should be used for the control of postoperative pain if indicated.
\end{abstract}

Key words: transurethral resection of prostate; pain anti-inflammatory drugs; paracetamol; analgesia Int Braz, J Urol. 2010; 36: 49-54

\section{INTRODUCTION}

Pain is a common symptom after endoscopic urologic surgery, and the need for effective pain management is obvious. Pain after TURP is due to bladder spasms and the catheter thus differs from open operations. The ideal postoperative analgesic treatment should provide rapid and effective pain relief, have a low incidence of adverse effects, and a minimal impact on organ systems or no significant interaction with other pharmacologic agents (1). Nonsteroidal anti-inflammatory drugs (NSAIDs) are commonly used for their potent antipyretic and analgesic effects. These drugs reduce pain after surgery by preventing the synthesis and release of prostaglandins at the site of surgical trauma by inhibition of cyclo-oxygenase2 (COX-2). COX-1 is found in most tissues under normal conditions. COX-2 is expressed in tissues that are traumatized by surgery or pathology within 2-12 hours after injury $(2,3)$. However, the use of 
NSAIDs for analgesia after surgery is controversial because NSAIDs, through antiplatelet activity by inhibition of the other isoform of cyclo-oxygenase, may increase the risk of postoperative bleeding. There are no available reported data concerning pain relief and postoperative bleeding effects of NSAIDs immediately after transurethral resection of the prostate.

The purpose of this prospective, randomized study was to compare the quality of analgesia and side-effects of parenteral NSAIDs versus parenteral paracetamol for postoperative analgesia after transurethral resection of the prostate.

\section{MATERIALS AND METHODS}

Fifty men with American Society of Anesthesiologists (ASA) physical status I or II, aged 55 to 75 years, undergoing transurethral resection of prostate (TURP) in our hospital were included in this study. Patients were excluded if they had a previous history of gastric or duodenal ulcer, allergy to NSAIDs or known severe systemic disease and using acetyl salicylic acid or finasteride. All patients were familiarized with a $10 \mathrm{~cm}$ visual analog scale (VAS) preoperatively with 0 : no pain and 10: the worse imaginable pain. Preoperative VAS scores were obtained from all patients. Patients were told to indicate the degree of pain by VAS, when they were asked to evaluate the intensity of their pain. Patients were divided randomly and prospectively into two groups (25 patients in each group), each routinely received either an NSAID (diclofenac) or paracetamol for postoperative analgesia. Group I (NSAID) received $75 \mathrm{mg}$ of diclofenac i.m. at the end of the operation followed by $75 \mathrm{mg}$ of diclofenac i.m. for 24 hours $(75 \mathrm{mg}$ x 2 once a day $=150 \mathrm{mg} / 24 \mathrm{~h}$ ) postoperatively. The other group (Group II) consisted of patients who received as postoperative analgesia $1 \mathrm{~g} / 100 \mathrm{~mL}$ i.v. paracetamol in 15 minutes twice daily. In case of inadequate analgesia (VAS score greater than 4), patients received meperidine i.m. $1 \mathrm{mg} / \mathrm{kg}$. Postoperative pain scores were evaluated at 30 minutes and 1,2, 4 and 6 hours after administration of each analgesic, using VAS. All adverse effects were recorded (e.g. nausea, vomiting, allergic reactions and headache). Preoperative and postoperative hemoglobin $(\mathrm{Hb})$ levels were recorded in all patients. Postoperative $\mathrm{Hb}$ measurements were performed on the evening of the operation and during the two postoperative days. In addition, hemostatic variables (bleeding time, prothrombin time and international normalized ratio ) were measured with $\mathrm{Hb}$. Statistical analysis was performed with Student's-ttest for quantitative data and the chi-square test for categorical data. A value of $p<0.05$ was considered statistically significant.

\section{RESULTS}

A total of 50 patients were randomized into two groups, parenteral diclofenac group (Group I, n $=25$ ) and intravenous paracetamol group (Group II, $\mathrm{n}=25$ ). Both groups (Group I and II) were similar with respect to age, prostate-specific antigen level, prostate volume measured by transrectal ultrasonography, body weight and height (Table-1). Moreover, no significant difference was observed between groups regarding the amount of resected tissue, operating time, preoperative-postoperative $\mathrm{Hb}$ levels and hemostatic variables (Table-1 and 2). In both groups, no patient required blood transfusion postoperatively. Postoperative adverse events for each group are recorded in Table-3 and they were similar between the two groups. Nausea, vomiting, injection site pain, pruritus and headache were reported adverse events. No respiratory depression, vertigo, ataxia, somnolence, hypotension and disorders in liver or kidney tests were observed in this study. Finally, pain score changes, during a 4 hour postoperative period

Table 1 -Demographic data of the patients in the group I and II. All values are median. No significant differences in any parameters $(p>0.05)$.

\begin{tabular}{lcc}
\hline & Group I & Group II \\
\hline Number & 25 & 25 \\
Age (year) & 66.8 & 64.3 \\
Weight $(\mathrm{kg})$ & 73.1 & 74.3 \\
Height $(\mathrm{cm})$ & 164.2 & 166.4 \\
TRUS volume $\left(\mathrm{cm}^{3}\right)$ & 63.8 & 61.0 \\
Operating time $(\mathrm{min})$ & 59.6 & 73.2 \\
\hline
\end{tabular}

TRUS = transrectal ultrasound 
Table 2 - Hemostatic parameters for the 2 groups. No significant differences in any parameters $(p>0.05)$.

\begin{tabular}{lcccccc}
\hline & \multicolumn{2}{c}{ Hemoglobin Values (g/dL) } & \multicolumn{2}{c}{ Prothrombine Time (sec) } & \multicolumn{2}{c}{ INR } \\
& Group I & Group II & Group I & Group II & Group I & Group II \\
\hline Preoperative & 13.9 & 14.1 & 13.1 & 12.9 & 1.1 & 1.1 \\
& $(10.8-17.2)$ & $(10.5-17.6)$ & $(11.4-14.2)$ & $(11.1-13.9)$ & $(0.9-1.2)$ & $(0.9-1.2)$ \\
Evening of day & 13.5 & 13.6 & 13.0 & 12.7 & 1.0 & 1.1 \\
& $(10.5-16.8)$ & $(10.2-17.3)$ & $(11.3-14.1)$ & $(11.2-13.7)$ & $(0.9-1.2)$ & $(0.8-1.2)$ \\
Postoperative day 1 & 13.4 & 13.6 & 13.2 & 12.8 & 1.0 & 1.0 \\
& $(10.5-16.6)$ & $(10.3-17.2)$ & $(11.4-14.1)$ & $(11.3-13.8)$ & $(0.8-1.1)$ & $(0.9-1.2)$ \\
Postoperative day 2 & 13.2 & 13.2 & 13.3 & 13.1 & 1.1 & 1.0 \\
& $(10.6-16.8)$ & $(10.6-16.8)$ & $(11.1-14.3)$ & $(11.5-13.9)$ & $(0.9-1.2)$ & $(0.8-1.2)$ \\
\hline
\end{tabular}

$I N R=$ international normalized ratio; that is the ratio of a patient's prothrombin time to a normal (control) sample.

between the two groups were similar ( $p=0.162$, Figure-1). Thirty minutes after surgery, pain scores were high $(>3 \mathrm{~cm})$ in both groups yet without any difference between them $(p=0.11)$ but 6 hours after surgery, pain scores were significantly higher with paracetamol compared with diclofenac $(\mathrm{p}<0.05)$. In the NSAID group, only 1 patient required additional analgesia with the administration of opiates, whereas in the paracetamol group 4 patients required such an additional postoperative analgesia. Bladder irrigation removal was routinely performed on postoperative day 1 in all patients. Urethral catheter was removed on postoperative day 2 or 3 (mean 2.7 days) and if the patient was comfortable, and afebrile, the patient was discharged home on the same day. There were no readmissions to the hospital.

\section{COMMENTS}

In this study, we demonstrate that the use of NSAIDs after TURP for analgesia is safe and effective. Besides their analgesic effects, anti-inflammatory properties of NSAIDs make them rational analgesics (4). Therefore, we performed our study with diclofenac, a NSAID, in patients undergoing TURP. Diclofenac was selected because it is readily accessible in our department and it is also easily administered to patients. Diclofenac has been successfully used in prevention and treatment of postoperative pain. One of the main reasons for avoiding NSAID consumption for postoperative pain is the fear to cause bleeding. NSAIDs are known for their tendency to cause bleeding, as a result of inhibition of cyclooxygenase and thrombocyte aggregation (4-6). Most urologists suggest the withdrawal of NSAIDs in patients undergoing TURP 7 to 10 days before the operation $(3,7)$. However, in our study we did not observe any difference in postoperative bleeding events between NSAID and the control group. This is consistent with previous studies that demonstrated a low incidence of postoperative bleeding with the use of NSAIDs when compared with narcotic analgesia after TURP (8-12). In a meta-analysis of 1,368 patients undergoing tonsillectomy, Krishna et al. reported that the incidence of postoperative bleeding was not affected by NSAID consumption (9). This finding was also confirmed by Moiniche et al. (10). In a post marketing study comparing 9,900 patients given ketorolac and

Table 3-Most frequently reports adverse events [number of patients (\%)].

\begin{tabular}{lcc}
\hline & Group I & Group II \\
\hline Nausea/vomiting & $2(8 \%)$ & $3(12 \%)$ \\
Injection site pain & $2(8 \%)$ & $0(0 \%)$ \\
Headache & $0(0 \%)$ & $1(4 \%)$ \\
Pruritus & $0(0 \%)$ & $1(4 \%)$ \\
\hline
\end{tabular}




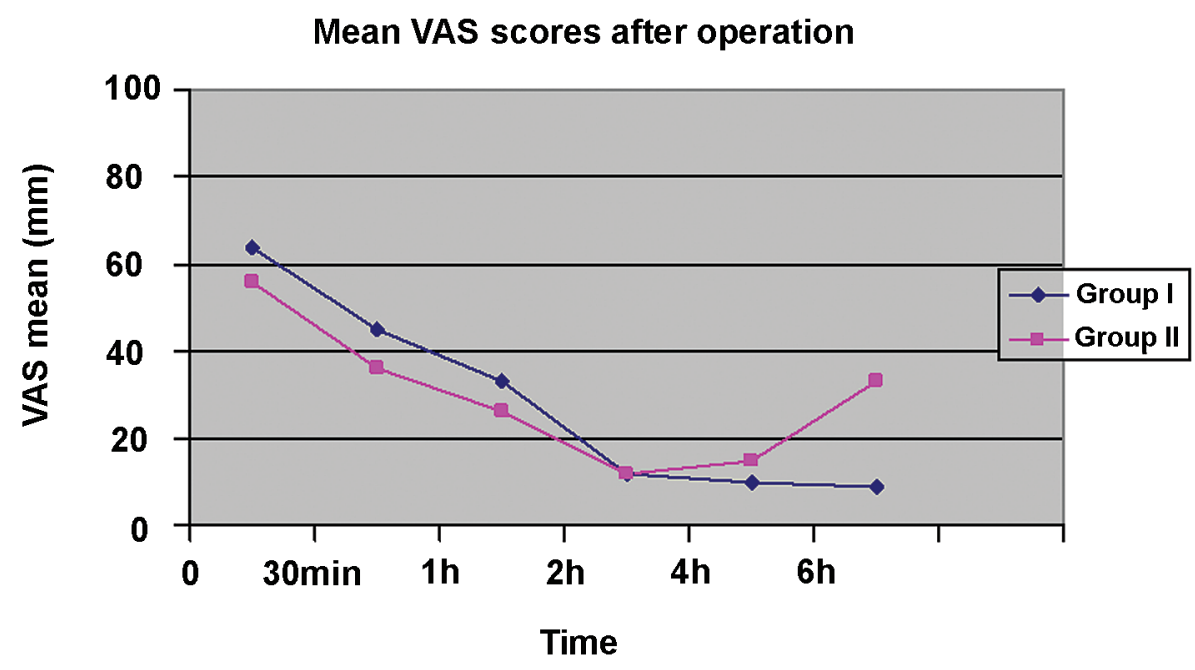

Figure 1 - The changes in postoperative visual analogue scale pain scores.

10,247 patients given an opioid, the only risk factors for operative site bleeding were age older than 75 year, dose higher than $100 \mathrm{mg}$, and treatment duration longer than 5 days (11). A subsequent subgroup analysis found no increase in the risk of clinically serious operative site bleeding among patients operated by otorhinolaryngologists (12).

Furthermore, our results show that 6 hours after prostate surgery performed under general anesthesia, a single dose NSAID alone is more effective for pain relief than a single infusion of paracetamol. Differences in pain scores were significant 6 hours after surgery. This result is in accordance with our knowledge on the analgesic properties of NSAIDs $(13,14)$. The ideal postoperative analgesic treatment should provide rapid and effective pain relief, have a low incidence of adverse effects, and minimal impact on major organ systems. NSAIDs have been shown to be as potent as opioids in adults and in children for major surgery $(13,15)$. Ehrlich et al. reported that early aspirin initiation after lower urinary tract surgery does not appear to carry an increased risk of postoperative bleeding. Thus, it may be considered in patients at high risk for cardiovascular morbidity (16). NSAIDs are not associated with increased incidence of nausea, vomiting, respiratory depression, decreased mental status and intestinal ileus compared with narcotics after surgery (17). Another potential advantage of using
NSAIDs is the reported decrease in the incidence of bladder spasms through the reduction of the amount of prostaglandins $(3,18,19)$. Moreover, NSAIDs may help reduce postoperative edema, resulting in more successful early catheter removal $(3,20)$. When we compared the drugs for cost effectiveness, the cost for the use of parenteral paracetamol was significantly higher. However, the aim of this study was not to demonstrate that the use of diclofenac is cost-effective but that NSAIDs could be a safe and effective alternative to other types of analgesics. Our study has some limitations. The number of participants was small, thus larger trials are required. Furthermore, in this study VAS scores were evaluated only for six hours. We think that randomized, double-blind, placebo-controlled trials are needed to further clarify the safety and efficacy of NSAIDs in the postoperative period with large patients groups.

\section{CONCLUSIONS}

There are no available data concerning pain relief and postoperative bleeding effects of NSAIDs immediately after transurethral resection of prostate. Our study shows that after TURP, the use of NSAIDs for postoperative analgesia is efficient for pain relief without an increased risk for bleeding. 


\section{CONFLICT OF INTEREST}

\author{
None declared.
}

\section{REFERENCES}

1. Sinatra RS, Jahr JS, Reynolds LW, Viscusi ER, Groudine SB, Payen-Champenois C: Efficacy and safety of single and repeated administration of 1 gram intravenous acetaminophen injection (paracetamol) for pain management after major orthopedic surgery. Anesthesiology. 2005; 102: 822-31.

2. Huang JJ, Taguchi A, Hsu H, Andriole GL Jr, Kurz A: Preoperative oral rofecoxib does not decrease postoperative pain or morphine consumption in patients after radical prostatectomy: a prospective, randomized, double-blinded, placebo-controlled trial. J Clin Anesth. 2001; 13: 94-7.

3. Mazaris EM, Varkarakis I, Chrisofos M, Skolarikos A, Ioannidis K, Dellis A, et al.: Use of nonsteroidal anti-inflammatory drugs after radical retropubic prostatectomy: a prospective, randomized trial. Urology. 2008; 72: 1293-7.

4. Arslan M, Tuncer B, Babacan A, Taneri F, Karadenizli Y, Onuk E, et al.: Postoperative analgesic effects of lornoxicam after thyroidectomy: a placebo controlled randomized study. Agri. 2006; 18: 27-33.

5. McCormack K: The evolving NSAID: focus on lornoxicam. Pain Rev. 1999; 6: 262-78.

6. Cooper SA, Hesch VE: Lornoxicam: Analgesic efficacy and safety of a new oxicam derivate. Advances in therapy. 1996; 13: 67-77.

7. Nielsen JD, Holm-Nielsen A, Jespersen J, Vinther CC, Settgast IW, Gram J: The effect of low-dose acetylsalicylic acid on bleeding after transurethral prostatectomy--a prospective, randomized, doubleblind, placebo-controlled study. Scand J Urol Nephrol. 2000; 34: 194-8.

8. Ala-Opas MY, Grönlund SS: Blood loss in long-term aspirin users undergoing transurethral prostatectomy. Scand J Urol Nephrol. 1996; 30: 203-6.

9. Krishna S, Hughes LF, Lin SY: Postoperative hemorrhage with nonsteroidal anti-inflammatory drug use after tonsillectomy: a meta-analysis. Arch Otolaryngol Head Neck Surg. 2003; 129: 1086-9.

10. Moiniche S, Romsing J, Dahl JB, Tramèr MR: Nonsteroidal antiinflammatory drugs and the risk of operative site bleeding after tonsillectomy: a quantitative systematic review. Anesth Analg. 2003; 96: 68-77.
11. Strom BL, Berlin JA, Kinman JL, Spitz PW, Hennessy S, Feldman H, et al.: Parenteral ketorolac and risk of gastrointestinal and operative site bleeding. A postmarketing surveillance study. JAMA. 1996; 275 : 376-82.

12. Gumprecht T: Risk of operative site bleeding with parenteral ketorolac. JAMA. 1996; 276: 372.

13. Fourcade O, Sanchez P, Kern D, Mazoit JX, Minville V, Samii K: Propacetamol and ketoprofen after thyroidectomy. Eur J Anaesthesiol. 2005; 22: 373-7.

14. Souter AJ, Fredman B, White PF: Controversies in the perioperative use of nonsterodial antiinflammatory drugs. Anesth Analg. 1994; 79: 1178-90.

15. Littlejohn IH, Tarling MM, Flynn PJ, Ordman AJ, Aiken A: Post-operative pain relief in children following extraction of carious deciduous teeth under general anaesthesia: a comparison of nalbuphine and diclofenac. Eur J Anaesthesiol. 1996; 13: 359-63.

16. Ehrlich Y, Yossepowitch O, Margel D, Lask D, Livne PM, Baniel J: Early initiation of aspirin after prostate and transurethral bladder surgeries is not associated with increased incidence of postoperative bleeding: a prospective, randomized trial. J Urol. 2007; 178: 524-8; discussion 528.

17. See WA, Fuller JR, Toner ML: An outcome study of patient-controlled morphine analgesia, with or without ketorolac, following radical retropubic prostatectomy. J Urol. 1995; 154: 1429-32.

18. Park JM, Houck CS, Sethna NF, Sullivan LJ, Atala A, Borer JG, et al.: Ketorolac suppresses postoperative bladder spasms after pediatric ureteral reimplantation. Anesth Analg. 2000; 91: 11-5.

19. Kanno T, Shibasaki N, Ito M, Tsuji Y, Taki Y, Takeuchi $\mathrm{H}$ : Early catheter removal and postoperative status of bladder outflow after retropubic radical prostatectomy. Hinyokika Kiyo. 2004; 50: 773-7.

20. Ead HM: Selective and nonselective nonsteroidal anti-inflammatory drugs in perianesthesia pain management. J Perianesth Nurs. 2008; 23: 335-41.

Accepted after revision: June 5, 2009 


\section{EDITORIAL COMMENT}

Nonsteroidal anti-inflammatory drugs (NSAIDs) are used frequently worldwide either because of their analgesic and anti-inflammatory properties or prophylactically for their antithrombotic activity. However, many urologists are reluctant to use NSAIDs before or after endoscopic and open operative procedures due to the increased possibility of hemorrhagic complications. In fact, randomized controlled trials supporting this view are actually lacking in the medical literature, thus there is not enough evidence to support such practice. On the contrary, there are initial results of small but welldesigned studies supporting the safety and efficacy of postoperative administration of NSAIDs after open radical prostatectomy (1) and after endoscopic prostate (TURP) or bladder surgery (2).

The authors of the present study are to be commended for their effort to assess the use of NSAIDs immediately after transurethral prostatectomy, for the first time in the medical literature. The aim of the study was to determine: 1) whether post-TURP patients on NSAIDs have the potential to bleed and 2) whether NSAIDs are effective in reducing postoperative pain after TURP. The study results confirmed the safety of NSAIDs immediately post-TURP although such patients may have the potential to bleed since vessels are either coagulated or expected to stop bleeding by catheter insertion and bladder irrigation. This is in contrast to open operations since bleeding vessels are either cauterized or ligated. The study was, however, underpowered to detect a difference in pain score in favor of NSAIDs although theoretically if larger studies are conducted with longer treatment times, they might prove to be advantageous. This is because post-TURP pain is mainly due to bladder spasms or catheter-related and NSAIDs have been found to have a positive effect on them through prostaglandin inhibition.

We agree with the authors that larger studies are worth being initiated in order to increase the evidence regarding safety and efficacy of NSAID administration after urological surgery.

\section{REFERENCES}

1. Mazaris EM, Varkarakis I, Chrisofos M, Skolarikos A, Ioannidis K, Dellis A, et al.: Use of nonsteroidal anti-inflammatory drugs after radical retropubic prostatectomy: a prospective, randomized trial. Urology. 2008; 72: 1293-7.

2. Ehrlich Y, Yossepowitch O, Margel D, Lask D, Livne PM, Baniel J: Early initiation of aspirin after prostate and transurethral bladder surgeries is not associated with increased incidence of postoperative bleeding: a prospective, randomized trial. J Urol. 2007; 178: 524-8; discussion 528.

Dr. Evangelos M. Mazaris \& Dr. Eleftherios Chatzidarellis 2nd Department of Urology Sismanoglio Hospital University of Athens Athens, Greece E-mail:evmazaris@yahoo.gr 